\title{
Noise Figure of a Balanced Amplifier
}

\author{
Akif Alperen Coskun ${ }^{\circledR}$, Student Member, IEEE, and Abdullah Atalar, Fellow, IEEE
}

\begin{abstract}
Noise figure expression of a balanced amplifier built with lossy divider and combiner and two imperfectly noisematched component amplifiers is derived analytically using the method of noise waves in terms of the noise parameters of the amplifiers. We present analytical expressions for the three noise parameters of the balanced amplifier. We show that a low-noise amplifier optimized to be used as a stand-alone amplifier is not the optimum choice for the component amplifiers of a balanced amplifier. The derived expressions can be used to choose or optimize the component amplifiers.
\end{abstract}

Index Terms-Balanced amplifiers, noise temperature, noise figure, noise parameters.

\section{INTRODUCTION}

A TYPICAL communications receiver's front end consists of a low-noise-amplifier (LNA) [1] preceded by a bandpass-filter to eliminate out-of-band signals. For proper operation, the filter requires a well-defined termination impedance, typically $50 \Omega$. On the other hand, the LNA's noise optimized (noise-matched) input impedance [2] is usually different than this value. Both requirements can be satisfied if two identical LNAs are combined in a balanced amplifier configuration, since a balanced amplifier provides a well-defined input impedance (typically $50 \Omega$ ) in a wide frequency band [3]. This configuration has the added benefit of higher linearity (or higher third-order intercept point) [4], [5]. Moreover, lossy networks needed in single amplifiers for stabilization purpose may be eliminated all together, since component amplifiers experience a 6-dB higher return loss from the source and load sides. A balanced amplifier has higher reliability, since the balanced amplifier will continue to work with a reduced performance when one of the component amplifiers fails. On the negative side, the addition of a power divider network at the input introduces extra loss and hence degrades the noise performance [6].

The noise performance of an ideal balanced amplifier was investigated and was shown to be independent of the phase of the source reflection coefficient [7]. The effects of the loss as well as the imbalances of the baluns on the noise figure of the balanced amplifier are investigated assuming the amplifiers have a perfectly matched input (infinite input return loss) [8].

While circuit simulators can be used to determine the noise performance for any circuit for a given source impedance, the

Manuscript received December 8, 2017; revised January 29, 2018; accepted January 29, 2018. Date of publication February 2, 2018; date of current version August 28, 2018. This brief was recommended by Associate Editor C.-T. Cheng. (Corresponding author: Akif Alperen Coskun.)

The authors are with the Department of Electrical and Electronics Engineering, Bilkent University, 06800 Ankara, Turkey (e-mail: alperen@ee.bilkent.edu.tr; aatalar@bilkent.edu.tr).

Color versions of one or more of the figures in this paper are available online at http://ieeexplore.ieee.org.

Digital Object Identifier 10.1109/TCSII.2018.2801759 results are difficult to interpret for optimizing the design. An analytical expression is more useful in that regard. An exact analytical expression for noise figure of a balanced amplifier was given in [9] for a special case (perfectly noise-matched amplifiers and lossless divider) without a derivation. The same work also gave the approximate noise figure expressions of the balanced amplifier using an imperfect divider with perfectly noise-matched amplifiers. In this brief, we provide analytical expressions of the noise figure and noise parameters for the case a balanced amplifier using imperfectly noise-matched amplifiers and a lossy divider, while presenting all the steps of the theoretical analysis using the noise wave method. We show that the expressions are useful in the optimization of amplifiers.

\section{Noise Figure of A StAnd-Alone Amplifier}

The noise figure of a stand-alone amplifier, $F_{A}$, can be written in terms of its source reflection coefficient, $\Gamma_{s}$, and its three noise parameters: the optimal source reflection coefficient, $\Gamma_{o}$, the minimum noise figure, $F_{m}$, and the normalized equivalent noise resistance, $r_{n} \cdot{ }^{1}$ When $\Gamma_{s}=\Gamma_{o}$, the noise figure equals $F_{m} . r_{n}$ is a factor showing how fast the noise figure degrades when $\Gamma_{s} \neq \Gamma_{o}$ given by [10]

$$
F_{A}=F_{m}+4 r_{n} \frac{\left|\Gamma_{s}-\Gamma_{o}\right|^{2}}{\left(1-\left|\Gamma_{s}\right|^{2}\right)\left|1+\Gamma_{o}\right|^{2}} .
$$

The three noise parameters are typically determined using noise figure measurements at least at four different source impedance values [11]. The accuracy can be improved, if more measurements [12] are taken. $^{2}$

Many amplifier manufacturers specify only the noise figure of an amplifier under $\Gamma_{s}=0$ condition, without specifying the noise parameters. Obviously, the noise figure alone is not sufficient information. If $\Gamma_{o}$ is zero, the noise figure depends on the magnitude of $\Gamma_{s}$, but not on its phase. On the other hand, if $\Gamma_{o}$ is not zero, the noise figure is also dependent on the phase of $\Gamma_{s}$. With given value of $\Gamma_{o}$, the worst case noise figure, $F_{\text {Amax }}$, is obtained, if $\Gamma_{S}$ and $\Gamma_{o}$ have a phase difference of $180^{\circ}$ :

$$
F_{\text {Amax }}=F_{m}+4 r_{n} \frac{\left(\left|\Gamma_{s}\right|+\left|\Gamma_{o}\right|\right)^{2}}{\left(1-\left|\Gamma_{s}\right|^{2}\right)\left|1+\Gamma_{o}\right|^{2}} .
$$

showing the importance of having $\left|\Gamma_{o}\right|=0$.

\footnotetext{
${ }^{1}$ For consistency, $r_{n} \geq\left(F_{m}-1\right) /\left(4 \operatorname{Re}\left\{\left(1-\Gamma_{o}\right) /\left(1+\Gamma_{o}\right)\right\}\right)$, must be satisfied. $\operatorname{Re}\{\}$ is the real part operator.

${ }^{2}$ Modern network analyzers find the noise parameters using seven measurements with seven known source impedances.
} 


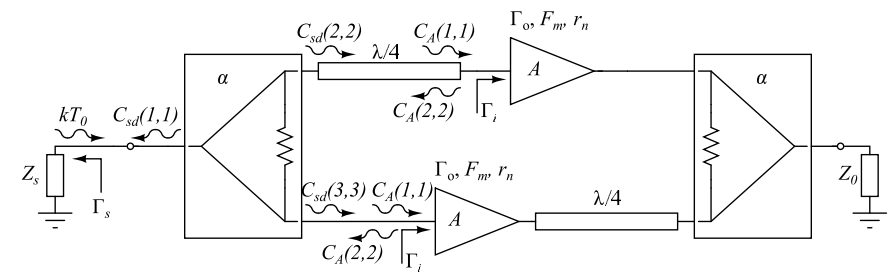

Fig. 1. A balanced amplifier built using 2 -way $0^{\circ}$ power dividers and two identical component amplifiers. Noise waves generated are also shown.

\section{Noise Figure of A BALANCEd Amplifier}

Suppose that we use two identical component amplifiers, two lossy 2 -way $0^{\circ}$ power dividers/combiners and two lossless quarter-wavelength transmission lines to build a balanced amplifier as depicted in Fig. 1. S-parameters of the unilateral amplifiers $\left(\mathbf{S}_{\mathbf{A}}\right)$ and the lossy two-way divider $\left(\mathbf{S}_{\mathbf{d}}\right)$ all specified with respect to $Z_{0}$ are assumed to be in the form

$$
\mathbf{S}_{\mathbf{A}}=\left[\begin{array}{cc}
\Gamma_{i} & 0 \\
A & 0
\end{array}\right], \mathbf{S}_{\mathbf{d}}=\sqrt{\frac{\alpha}{2}}\left[\begin{array}{ccc}
0 & -j & -j \\
-j & 0 & 0 \\
-j & 0 & 0
\end{array}\right]
$$

where $-10 \log (\alpha)$ is the ohmic loss of the divider expressed in $\mathrm{dB}$. A real-life power divider has finite isolation and imperfect match at its ports and it may have an amplitude and a phase imbalance. All these parameters have small but statistically random amplitude and phase, varying from sample to sample. Hence it is difficult to model a real power divider for the purpose of optimizing the balanced amplifier noise figure. We determined the full expression of noise figure [9] for the most general case using a symbolic computational package, but it is too long to be useful as a guidance. ${ }^{3}$ We prefer to approximate the divider in the idealized form of (3) for the purpose of obtaining a simple analytical expression useful for design. Besides, attenuation $(\alpha)$ is the only accurate parameter of a real divider.

To find an analytical expression for the noise figure, $F_{b}$, of a balanced amplifier, we determine the total available output noise power in $1 \mathrm{~Hz}$ bandwidth $\left(N_{o}\right)$ and divide it with available output noise power caused by the available noise from the source $\left(N_{S}\right)$ in the same bandwidth:

$$
F_{b}=\left.\frac{N_{o}}{G_{A} N_{s}}\right|_{N_{s}=k T_{0}}
$$

where $G_{A}$ is the available gain of the amplifier. The noise temperature of the source impedance must be kept at the standard noise temperature of $T_{0}=290^{\circ} \mathrm{K} .{ }^{4}$ We assume that the available gain of the amplifier, $G_{A}$, is sufficiently high so that the output combiner does not contribute significantly to the output noise. To determine the output noise we utilize the method of noise waves [10], [13], [14].

A noisy $n$-port network can be replaced with the noiseless version of the network and outgoing noise waves $\left(b_{n i}\right.$ 's) from

\footnotetext{
${ }^{3}$ Numerical evaluation of that expression showed that amplitude or phase balance of divider have a small effect on the noise figure.

${ }^{4} F=S N R_{\text {in }} / S N R_{\text {out }}$ is a more common definition used in communication textbooks. Many textbooks neglect to mention the condition that the input noise temperature should be at $T_{0}=290^{\circ} \mathrm{K}$, leading to errors or confusion among students and unexperienced engineers.
}

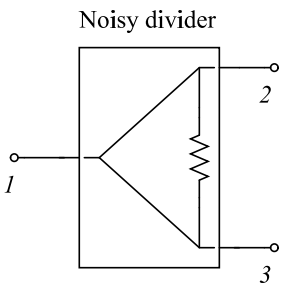

(a)

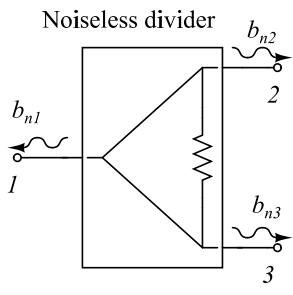

(b)
Fig. 2. A noisy divider (a) is equivalent to a noiseless version of divider with outgoing noise waves at all its ports (b).

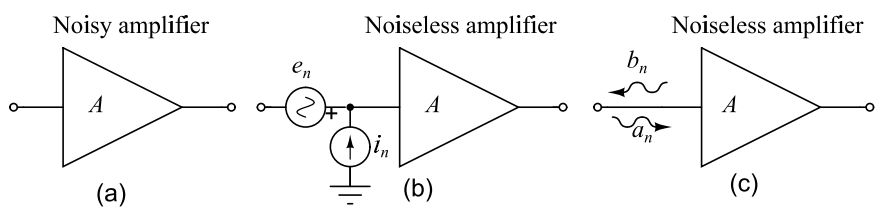

Fig. 3. A noisy amplifier (a) is equivalent to a noise-free amplifier with a voltage and a current noise source at its input (b) or that with two noise waves at its input (c).

all its ports as demonstrated in Fig. 2 for a 2-way divider. When the network is passive with internal components at the standard noise temperature of $T_{0}$, the correlation matrix of noise waves, $\mathbf{C}_{\mathbf{s}}$, can be written as [15]

$$
\mathbf{C}_{\mathbf{s}}=k T_{0}\left(\mathbf{I}-\mathbf{S S}^{\dagger}\right)
$$

where $\mathbf{S}$ is the $\mathbf{S}$-parameter matrix of the $n$-port and the dagger indicates the conjugate transpose operation.

For the case of 2-way divider, the correlation matrix of the noise waves, $\mathbf{C}_{\mathbf{s d}}$, is found from (5) as

$$
\begin{aligned}
& \mathbf{C}_{\mathbf{s d}}=\left[\begin{array}{ccc}
\left|b_{n 1}\right|^{2} & b_{n 1} b_{n 2}^{*} & b_{n 1} b_{n 3}^{*} \\
b_{n 1}^{*} b_{n 2} & \left|b_{n 2}\right|^{2} & b_{n 2} b_{n 3}^{*} \\
b_{n 1}^{*} b_{n 3} & b_{n 2}^{*} b_{n 3} & \left|b_{n 3}\right|^{2}
\end{array}\right] \\
&=k T_{0}\left(\mathbf{I}-\mathbf{S}_{\mathbf{d}} \mathbf{S}_{\mathbf{d}}^{\dagger}\right)=k T_{0}\left[\begin{array}{ccc}
1-\alpha & 0 & 0 \\
0 & 1-\frac{\alpha}{2} & -\frac{\alpha}{2} \\
0 & -\frac{\alpha}{2} & 1-\frac{\alpha}{2}
\end{array}\right] .
\end{aligned}
$$

Here, diagonal elements show the power of the noise waves, while the off-diagonal elements represent the correlation between the waves.

A noisy amplifier can be represented by a noise-free amplifier and two (partially correlated) noise sources at its input: A voltage noise source of $e_{n}$ and a current noise source of $i_{n}$ as shown in Fig. 3(b). These noise sources can be written in terms of the noise parameters of the amplifier in the form of a correlation matrix as [16]

$$
\left[\begin{array}{cc}
\left|e_{n}\right|^{2} & e_{n} i_{n}^{*} \\
e_{n}^{*} i_{n} & \left|i_{n}\right|^{2}
\end{array}\right]=4 k T_{0}\left[\begin{array}{cc}
r_{n} & \frac{F_{m}-1}{2}-r_{n} \bar{Y}_{o}^{*} \\
\frac{F_{m}-1}{2}-r_{n} \bar{Y}_{o} & r_{n}\left|\bar{Y}_{o}\right|^{2}
\end{array}\right]
$$

where $\bar{Y}_{o}=\left(1-\Gamma_{o}\right) /\left(1+\Gamma_{o}\right)$ is the normalized optimal source admittance. The noise sources can be replaced with incoming and outgoing noise waves (See Fig. 3(c)) at the input of the amplifier represented by $a_{n}$ and $b_{n}$ (with $Z_{0}=1$ ) [13]

$$
a_{n}=-\frac{e_{n}+i_{n}}{2} \text { and } \quad b_{n}=\frac{e_{n}-i_{n}}{2} .
$$




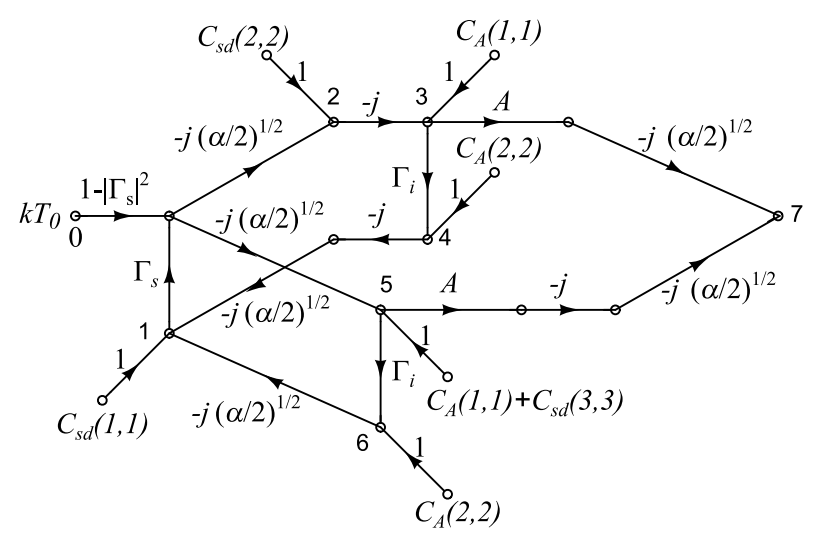

Fig. 4. Signal flow graph of the balanced amplifier showing the noise wave excitations.

Hence, we can write the correlation matrix in wave representation as

$$
\begin{aligned}
\mathbf{C}_{\mathbf{A}} & =\left[\begin{array}{cc}
\left|a_{n}\right|^{2} & a_{n} b_{n}^{*} \\
a_{n}^{*} b_{n} & \left|b_{n}\right|^{2}
\end{array}\right] \\
& =k T_{0}\left[\begin{array}{cc}
r_{n}\left|1-\bar{Y}_{o}\right|^{2}+F_{m}-1 & -r_{n}\left(1-\left|\bar{Y}_{o}\right|^{2}-2 j b_{o}\right) \\
-r_{n}\left(1-\left|\bar{Y}_{o}\right|+2 j b_{o}\right) & r_{n}\left|1+\bar{Y}_{o}\right|^{2}-F_{m}+1
\end{array}\right] \\
& =k T_{0}\left[\begin{array}{cc}
\frac{4 r_{n}\left|\Gamma_{o}\right|^{2}}{\left|1+\Gamma_{o}\right|^{2}}+F_{m}-1 & -\frac{4 r_{n} \Gamma_{o}}{\left|1+\Gamma_{o}\right|^{2}} \\
-\frac{4 r_{n} \Gamma_{o}^{*}}{\left|1+\Gamma_{o}\right|^{2}} & \frac{4 r_{n}}{\left|1+\Gamma_{o}\right|^{2}}-F_{m}+1
\end{array}\right]
\end{aligned}
$$

If $\Gamma_{o}=0$, the off-diagonal terms in $C_{A}$ are zero, indicating that $a_{n}$ and $b_{n}$ are uncorrelated. On the other hand, for $\Gamma_{o} \neq 0$, there is a correlation between these noise waves.

A signal flow graph [17] of the balanced amplifier is given in Fig. 4. The quantities at the excitation nodes correspond to the available powers of noise waves.

The available gain of the balanced amplifier between input (node 0) and output (node 7) is given by

$$
G_{A}=\alpha^{2}|A|^{2}\left(1-\left|\Gamma_{s}\right|^{2}\right)
$$

which includes the effect of source impedance mismatch.

Transfer function, $T_{i}$, from the excitation node $i$ to the output node 7 are found by Mason's rule [18]:

$$
\begin{aligned}
& T_{1}=j \Gamma_{s} \alpha A, \\
& T_{2}=-j T_{3}=-\left(1+\alpha \Gamma_{s} \Gamma_{i}\right) \sqrt{\frac{\alpha}{2}} A, \\
& T_{4}=-j T_{6}=-j \Gamma_{s} \alpha \sqrt{\frac{\alpha}{2}} A, \\
& T_{5}=-\left(1-\alpha \Gamma_{s} \Gamma_{i}\right) \sqrt{\frac{\alpha}{2}} A .
\end{aligned}
$$

We find the noise power contributions of six noise sources at the output as [14]

$$
\begin{aligned}
N_{1} & =C_{s d}(1,1)\left|T_{1}\right|^{2}=k T_{0}(1-\alpha) \alpha^{2}\left|\Gamma_{s} A\right|^{2}, \\
N_{2} & =C_{s d}(2,2)\left|T_{2}\right|^{2}=k T_{0}\left(1-\frac{\alpha}{2}\right)\left|1+\alpha \Gamma_{s} \Gamma_{i}\right|^{2} \frac{\alpha}{2}|A|^{2}, \\
N_{3} & =C_{A}(1,1)\left|T_{3}\right|^{2} \\
& =k T_{0}\left(\frac{4 r_{n}\left|\Gamma_{o}\right|^{2}}{\left|1+\Gamma_{o}\right|^{2}}+F_{m}-1\right)\left|1+\alpha \Gamma_{s} \Gamma_{i}\right|^{2} \frac{\alpha}{2}|A|^{2}
\end{aligned}
$$

$$
\begin{aligned}
N_{4} & =N_{6}=C_{A}(2,2)\left|T_{4}\right|^{2} \\
& =k T_{0}\left(\frac{4 r_{n}}{\left|1+\Gamma_{o}\right|^{2}}-F_{m}+1\right)\left|\Gamma_{s}\right|^{2} \frac{\alpha^{3}}{2}|A|^{2}, \\
N_{5} & =\left(C_{s d}(3,3)+C_{A}(1,1)\right)\left|T_{5}\right|^{2} \\
& =k T_{0}\left(\frac{4 r_{n}\left|\Gamma_{o}\right|^{2}}{\left|1+\Gamma_{o}\right|^{2}}+F_{m}-\frac{\alpha}{2}\right)\left|1-\alpha \Gamma_{s} \Gamma_{i}\right|^{2} \frac{\alpha}{2}|A|^{2} .
\end{aligned}
$$

Since some noise sources are correlated, we need to include the cross-correlation terms as well [14]. These are

$$
\begin{aligned}
N_{25} & =C_{s d}(2,3) T_{2} T_{5}^{*}+C_{s d}(3,2) T_{5} T_{2}^{*} \\
& =-k T_{0}\left(1-\alpha^{2}\left|\Gamma_{s}\right|^{2}\left|\Gamma_{i}\right|^{2}\right) \frac{\alpha^{2}|A|^{2}}{2}, \\
N_{34} & =C_{A}(1,2) T_{3} T_{4}^{*}+C_{A}(2,1) T_{4} T_{3}^{*} \\
& =-k T_{0} \frac{4 r_{n} \alpha^{2}|A|^{2}}{\left|1+\Gamma_{o}\right|^{2}} \operatorname{Re}\left\{\left(1+\alpha \Gamma_{s} \Gamma_{i}\right) \Gamma_{s}^{*} \Gamma_{o}\right\}, \\
N_{56} & =C_{A}(1,2) T_{5} T_{6}^{*}+C_{A}(2,1) T_{6} T_{5}^{*} \\
& =k T_{0} \frac{4 r_{n} \alpha^{2}|A|^{2}}{\left|1+\Gamma_{o}\right|^{2}} \operatorname{Re}\left\{\left(1-\alpha \Gamma_{s} \Gamma_{i}\right) \Gamma_{s}^{*} \Gamma_{o}\right\} .
\end{aligned}
$$

Using the identities

$$
\begin{aligned}
|1+z|^{2}+|1-z|^{2} & =2\left(1+|z|^{2}\right), \\
1-2 \operatorname{Re}\{z\}+|z|^{2} & =|1-z|^{2},
\end{aligned}
$$

and (15)-(22), the noise figure, $F_{b}=1+\sum N /\left(k T_{0} G_{A}\right)$, of the balanced amplifier can be written as

$$
\begin{array}{r}
F_{b}=\frac{1}{\alpha\left(1-\left|\Gamma_{s}\right|^{2}\right)}\left(F_{m}\left[1-\alpha^{2}\left|\Gamma_{s}\right|^{2}\left(1-\left|\Gamma_{i}\right|^{2}\right)\right]\right. \\
\left.+4 r_{n} \frac{\left|\Gamma_{o}\right|^{2}+\alpha^{2}\left|\Gamma_{s}\right|^{2}\left|1-\Gamma_{i} \Gamma_{o}\right|^{2}}{\left|1+\Gamma_{o}\right|^{2}}\right)
\end{array}
$$

For the special case of $\Gamma_{i}=\Gamma_{o}=0^{5}$ and a lossless divider $(\alpha=1)$, the noise figure expression of (25) reduces to

$$
F_{b}=F_{m}+4 r_{n} \frac{\left|\Gamma_{s}\right|^{2}}{1-\left|\Gamma_{s}\right|^{2}}
$$

which is the same as the noise figure expression of the single amplifier given in (1) with $\Gamma_{o}=0$. If the divider is lossless $(\alpha=1)$, the amplifiers are perfectly noise-matched $\left(\Gamma_{o}=\right.$ $0)$ and but the amplifier input impedances are not matched $\left(\Gamma_{i} \neq 0\right), F_{b}$ is equivalent to the noise figure expression given in [9]:

$$
F_{b}=F_{m}+4\left(r_{n}+F_{m} \frac{\left|\Gamma_{i}\right|^{2}}{4}\right) \frac{\left|\Gamma_{s}\right|^{2}}{1-\left|\Gamma_{s}\right|^{2}}
$$

\section{Noise PARAmeters of the \\ BALANCED AMPLIFIER}

By rearranging (25), we can find the three noise parameters of the balanced amplifier, $\Gamma_{o b}, F_{m b}$ and $r_{n b}$, as

$$
\begin{aligned}
\Gamma_{o b} & =0 \\
F_{m b} & =\frac{1}{\alpha}\left(F_{m}+4 r_{n} \frac{\left|\Gamma_{o}\right|^{2}}{\left|1+\Gamma_{o}\right|^{2}}\right)
\end{aligned}
$$

${ }^{5}$ Not a realistic assumption, since noise-matching and input-matching of amplifiers cannot be attained simultaneously. 
TABLE I

NoIse Figure AND NoIse PARAMETer COMPARISON

\begin{tabular}{|c||c|c|c|}
\hline$F_{m}(\mathrm{~dB})$ & 1.0 & 1.5 & 2.5 \\
\hline$r_{n}$ & 0.1 & 0.35 & 0.5 \\
\hline$\Gamma_{o}$ & $-0.1+j 0.2$ & $0.3-j 0.55$ & $0.4+j 0.12$ \\
\hline$\Gamma_{i}$ & $-0.1-j 0.2$ & $-0.2+j 0.4$ & $0.4-j 0.3$ \\
\hline$\alpha(\mathrm{dB})$ & -0.2 & -0.3 & -0.5 \\
\hline$\Gamma_{s}$ & $0.4-j 0.2$ & $0.5+j 0.6$ & $0.7-j 0.6$ \\
\hline$F_{b}(\mathrm{~dB})(25)$ & 1.74489747 & 5.76004409 & 10.5002865 \\
\hline$F_{b}(\mathrm{~dB})(\mathrm{AWR})$ & 1.7448974 & 5.7600441 & 10.5002865 \\
\hline$F_{b}(\mathrm{~dB})(\mathrm{ADS})$ & 1.7448976 & 5.760044 & 10.5002865 \\
\hline$F_{m b}(\mathrm{~dB})(29)$ & 1.28042075 & 2.574552116 & 3.411335826 \\
\hline$F_{m b}(\mathrm{~dB})(\mathrm{AWR})$ & 1.2804207 & 2.57455212 & 3.41133583 \\
\hline$F_{m b}(\mathrm{~dB})(\mathrm{ADS})$ & 1.2804207 & 2.57455211 & 3.41133588 \\
\hline$r_{n b}(\mathrm{~dB})(30)$ & 0.151583703 & 0.312960119 & 0.398269643 \\
\hline$r_{n b}(\mathrm{~dB})(\mathrm{AWR})$ & 0.1515837 & 0.312960 & 0.39826964 \\
\hline$r_{n b}(\mathrm{~dB})(\mathrm{ADS})$ & 0.1515837 & 0.312960 & 0.39826964 \\
\hline
\end{tabular}

$$
r_{n b}=\frac{F_{m}}{4 \alpha}\left(1-\alpha^{2}\left(1-\left|\Gamma_{i}\right|^{2}\right)\right)+\frac{r_{n}}{\alpha} \frac{\left|\Gamma_{o}\right|^{2}+\alpha^{2}\left|1-\Gamma_{i} \Gamma_{o}\right|^{2}}{\left|1+\Gamma_{o}\right|^{2}}
$$

Hence we have

$$
F_{b}=F_{m b}+4 r_{n b} \frac{\left|\Gamma_{s}\right|^{2}}{\left(1-\left|\Gamma_{s}\right|^{2}\right)} .
$$

Equation (28) indicates that the optimal source impedance for a balanced amplifier is $Z_{0}$ and the noise figure $F_{b}$ is independent of $\Gamma_{s}$ phase [7]. This is consistent with the approximate formula given earlier [9]: $\Gamma_{o b} \approx S_{11}^{*}$ where $S_{11}$ is the reflection coefficient of the divider at port 1 , which in this analysis is assumed to be zero.

For the purpose of verification of the formulas, two commercial circuit simulators ${ }^{6}$ are used. Table I is a comparison ${ }^{7}$ of the results obtained for three cases.

A balanced amplifier can also be built using a 3-dB hybrid coupler (or a Lange coupler) with a wider bandwidth. The results given are also valid for such a balanced amplifier, if the S-parameters of the lossy hybrid coupler with a perfectly terminated fourth port can be defined as a three-port network:

$$
\mathbf{S}_{\mathbf{c}}=\sqrt{\frac{\alpha}{2}}\left[\begin{array}{ccc}
0 & -j & -1 \\
-j & 0 & 0 \\
-1 & 0 & 0
\end{array}\right]
$$

\section{Discussion}

Since the terms $\left|1+\Gamma_{o}\right|^{2}$ and $\left|1-\Gamma_{i} \Gamma_{o}\right|^{2}$ of (29) and (30) depend on the phases of the reflection coefficients, the two noise parameters are also dependent on the phases of $\Gamma_{i}$ and $\Gamma_{o}$. When $\Gamma_{o}$ gets larger, $F_{m b}$ is degraded and $r_{n b}$ is increased for most cases, especially if $\Gamma_{i}$ is large. Inspection of (29) and (30) indicates that keeping $\Gamma_{o}$ real and positive and $\angle \Gamma_{i}=$ $-\angle \Gamma_{o}$ is the optimal choice for the noise performance.

Fig. 5 shows the range of noise figures of a balanced amplifier as a function of $\left|\Gamma_{s}\right|,\left|\Gamma_{o}\right|$ and $\left|\Gamma_{i}\right|$, as the phase of these variables are varied. The noise figure increases as these variables are increased. The same graphs also show the limits of

\footnotetext{
${ }^{6}$ AWR (v. 12) from AWR Corp. (http://www.awrcorp.com) and ADS (v. 2016.01) from Keysight Technologies (www.keysight.com).

${ }^{7}$ When $\Gamma_{s}$ is a complex number, ADS calculates $F_{m}$ and $r_{n}$ values incorrectly, while giving the correct result for $F_{b}$. We used $\Gamma_{s}=\left|\Gamma_{s}\right|$ for ADS to calculate $F_{m}$ and $r_{n}$.
}
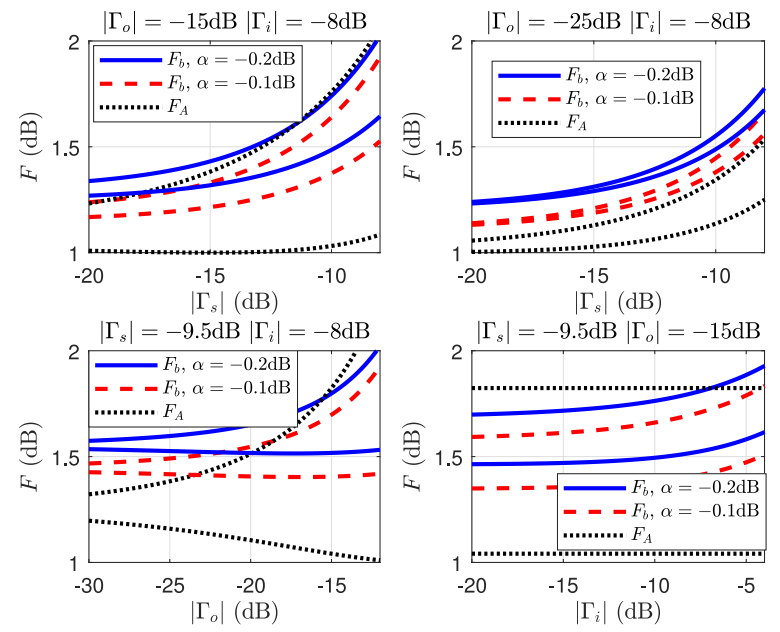

Fig. 5. Maximum and minimum noise figures of balanced amplifiers as a function of $\left|\Gamma_{s}\right|,\left|\Gamma_{o}\right|$ and $\left|\Gamma_{i}\right|$, as the phase of all complex variables are varied. Component amplifiers have $F_{m}=1 \mathrm{~dB}, r_{n}=0.15$. The resulting range of noise figure for the component amplifiers is also shown for comparison.

the noise figure for a single amplifier when the phase of $\Gamma_{S}$ is changed. In spite of the lossy divider, the noise figure for a balanced amplifier can be smaller than that for the component amplifier, if $\left|\Gamma_{o}\right|$ is large or if $\left|\Gamma_{i}\right|$ is small.

The expressions (29)-(31) are approximately true also for imperfect dividers as long as $\left|S_{11}\right|$ of the divider is small. An example simulation of an imperfect divider centered at 1.8 GHz is shown in Fig. 6, which is used to build a balanced amplifier. The component amplifiers operating at the same frequency utilize the low-noise PHEMT transistor ATF-38143 giving a gain of $14 \mathrm{~dB}$. Fig. 7 displays the simulation results for the relevant parameters of component amplifiers. The same figure also compares the noise figure and noise parameters of the balanced amplifier as obtained from the simulator and the analytical expressions. The worst case noise figure defined by

$$
F_{\text {bmax }}=F_{m b}+4 r_{n b} \frac{\left(\left|\Gamma_{s}\right|+\left|\Gamma_{o b}\right|\right)^{2}}{\left(1-\left|\Gamma_{s}\right|^{2}\right)\left|1+\Gamma_{o b}\right|^{2}}
$$

with exact values found by simulator is also plotted. $F_{m b}$ and $r_{n b}$ are estimated well. With an imperfect divider we have the approximation $\Gamma_{o b} \approx S_{11}^{*}$ [9], hence $\Gamma_{o b}$ is not exactly zero, resulting in more error in $F_{b}$.

If a balanced amplifier uses component amplifiers with minimized $F_{\text {Amax }}$ given by (2), an optimum $F_{b}$ is not obtained, since the minimization of $\Gamma_{i}$ is disregarded. $F_{b}$ can be optimized using (31) as the goal function to be minimized during the design of component amplifiers. One should use the expected value of $\alpha$ and $\left|\Gamma_{s}\right|$ (e.g., $\left.-9.5 \mathrm{~dB}\right)$ in the expression. This will ensure that both $\Gamma_{o}$ and $\Gamma_{i}$ are minimized with the correct weighting. The proposed approach is the best that can be done even when an imperfect divider is used, since the small amplitudes and random phases of remaining parameters of the divider can not be specified. The worst case noise figure, $F_{b \max }$, of the balanced amplifier given by (33) using an imperfect divider will be somewhat larger than the noise figure predicted by (31). Using a better divider with a small $\left|S_{11}\right|$ reduces the difference. 

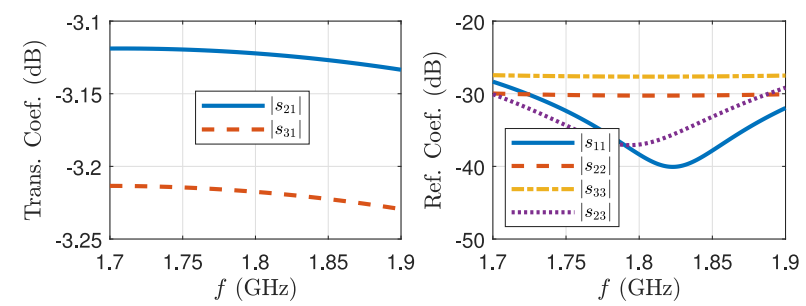

Fig. 6. Characteristics of the example lossy divider with an amplitude imbalance. It has an ohmic loss of $\alpha=-0.17 \mathrm{~dB}$.
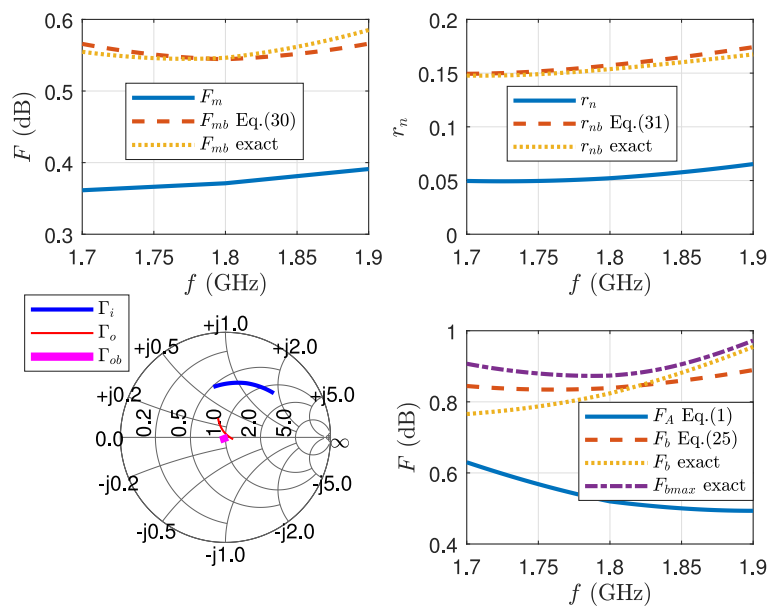

Fig. 7. Comparison of analytical and simulated results for the example balanced amplifier using the divider of Fig. 6 . The noise parameters for the component amplifiers are also shown. $F_{A}, F_{b}$ with $\Gamma_{s}=-9.5 \mathrm{~dB} \angle 180^{\circ}$, $F_{\text {bmax }}$ with $\left|\Gamma_{s}\right|=-9.5 \mathrm{~dB}$.

\section{CONCLUSION}

We analyze the noise performance for a balanced amplifier and provide analytical expressions for the noise figure and noise parameters. We find that a balanced amplifier is more forgiving to imperfect noise-matching of the component amplifiers or to higher source mismatch. An amplifier optimized to be used as a stand-alone LNA may not be the most suitable choice as a component amplifier of a balanced amplifier. While a non-zero input reflection $\left(\Gamma_{i}\right)$ does not influence the noise performance of a stand-alone amplifier, it negatively affects the noise figure of a balanced amplifier.

Since the amplitude and phase of near-zero parameters of a real divider are random and unknown, using such a divider in a simulator for the optimization is not possible. Since $F_{b} \leq F_{b \max }$, the noise figure expression obtained for the idealized divider defines a lower limit for the worst case noise figure of a balanced amplifier using an imperfect divider. Using the expression (31) at the intended $\left|\Gamma_{s}\right|$ as the function to be minimized in a simulator is the optimal approach to design or choose a component amplifier. To reduce the degradation in the noise figure, a high performance divider (or a hybrid coupler) with low loss and good matching at the ports - especially at the input port, should be used.

\section{REFERENCES}

[1] A. Amer, E. Hegazi, and H. Ragai, "A low-power wideband CMOS LNA for WiMAX," IEEE Trans. Circuits Syst. II, Exp. Briefs, vol. 54, no. 1 , pp. 4-8, Jan. 2007.

[2] K.-C. He, M.-T. Li, C.-M. Li, and J.-H. Tarng, "Parallel-RC feedback low-noise amplifier for UWB applications," IEEE Trans. Circuits Syst. II, Exp. Briefs, vol. 57, no. 8, pp. 582-586, Aug. 2010.

[3] J. Zhu and P. R. Kinget, "Frequency-translational quadrature-hybrid receivers for very-low-noise, frequency-agile, scalable inter-band carrier aggregation," IEEE J. Solid-State Circuits, vol. 51, no. 12, pp. 3137-3151, Dec. 2016.

[4] I. Malo-Gómez, J. D. Gallego-Puyol, C. Diez-González, I. López-Fernández, and C. Briso-Rodríguez, "Cryogenic hybrid coupler for ultra-low-noise radio astronomy balanced amplifiers," IEEE Trans. Microw. Theory Techn., vol. 57, no. 12, pp. 3239-3245, Dec. 2009.

[5] P. V. Testa et al., "A 210-GHz SiGe balanced amplifier for ultrawideband and low-voltage applications," IEEE Microw. Wireless Compon. Lett., vol. 27, no. 3, pp. 287-289, Mar. 2017.

[6] S. Seo, D. Pavlidis, and J. S. Moon, "Wideband balanced AlGaN/GaN HEMT MMIC low noise amplifier," Electron. Lett., vol. 41, no. 16, pp. 909-911, Aug. 2005.

[7] A. R. Kerr, "On the noise properties of balanced amplifiers," IEEE Microw. Guided Wave Lett., vol. 8, no. 11, pp. 390-392, Nov. 1998.

[8] Ó. Garcia-Pérez, V. González-Posadas, L. E. García-Muñoz, and D. Segovia-Vargas, "Noise-figure measurement of differential amplifiers using nonideal baluns," IEEE Trans. Microw. Theory Techn., vol. 59, no. 6, pp. 1658-1664, Jun. 2011.

[9] A. A. Coskun and A. Atalar, "Noise figure degradation in balanced amplifiers," IEEE Microw. Wireless Compon. Lett., vol. 27, no. 9, pp. 848-850, Sep. 2017.

[10] R. P. Meys, "A wave approach to the noise properties of linear microwave devices," IEEE Trans. Microw. Theory Techn., vol. MTT-26, no. 1, pp. 34-37, Jan. 1978.

[11] G. I. Vasilescu, G. Alquie, and M. Krim, "Exact computation of two-port noise parameters," Electron. Lett., vol. 25, no. 4, pp. 292-293, Feb. 1989.

[12] J. M. O'Callaghan and J. P. Mondal, "A vector approach for noise parameter fitting and selection of source admittances," IEEE Trans. Microw. Theory Techn., vol. 39, no. 8, pp. 1376-1382, Aug. 1991.

[13] P. Penfield, "Wave representation of amplifier noise," IRE Trans. Circuit Theory, vol. 9, no. 1, pp. 84-86, Mar. 1962.

[14] N. G. Kanaglekar, R. E. McIntosh, and W. E. Bryant, "Wave analysis of noise in interconnected multiport networks," IEEE Trans. Microw. Theory Techn., vol. MTT-35, no. 2, pp. 112-116, Feb. 1987.

[15] S. W. Wedge and D. B. Ruthledge, "Wave techniques for noise modeling and measurement," IEEE Trans. Microw. Theory Techn., vol. 40, no. 11, pp. 2004-2012, Nov. 1992.

[16] L. Escotte, R. Plana, and J. Graffeuil, "Evaluation of noise parameter extraction methods," IEEE Trans. Microw. Theory Techn., vol. 41, no. 3, pp. 382-387, Mar. 1993.

[17] J. K. Hunton, "Analysis of microwave measurement techniques by means of signal flow graphs," IRE Trans. Microw. Theory Techn., vol. 8, no. 2, pp. 206-212, Mar. 1960.

[18] S. J. Mason, "Feedback theory-further properties of signal flow graphs," Proc. IRE, vol. 44, no. 7, pp. 920-926, Jul. 1956. 